\title{
Combining Color Features for Real-Time Correlation Tracking
}

\author{
Yulong $\mathrm{XU}^{\dagger}$, Zhuang MIAO ${ }^{\dagger a)}$, Jiabao WANG ${ }^{\dagger}$, Yang $\mathrm{LI}^{\dagger}$, Nonmembers, Hang $\mathrm{LI}^{\dagger}$, Member, \\ Yafei ZHANG ${ }^{\dagger}$, Weiguang $\mathrm{XU}^{\dagger}$, Nonmembers, and Zhisong PAN ${ }^{\dagger}$, Member
}

\begin{abstract}
SUMMARY Correlation filter-based approaches achieve competitive results in visual tracking, but the traditional correlation tracking methods failed in mining the color information of the videos. To address this issue, we propose a novel tracker combined with color features in a correlation filter framework, which extracts not only gray but also color information as the feature maps to compute the maximum response location via multichannel correlation filters. In particular, we modify the label function of the conventional classifier to improve positioning accuracy and employ a discriminative correlation filter to handle scale variations. Experiments are performed on 35 challenging benchmark color sequences. And the results clearly show that our method outperforms state-of-the-art tracking approaches while operating in real-time.

key words: correlation filter, color feature, real-time visual tracking
\end{abstract}

\section{Introduction}

Visual object tracking is one of the active research topics in the field of computer vision, which plays an important role in many applications. In visual tracking, the task is to estimate the locations of a target in an image sequence by giving only its initial position. This is especially challenging due to several factors such as scale changes, illumination variations, fast motion, motion blur, rotation, occlusion and background clutter [1], [2].

In recent years, the correlation filter-based approaches [3]-[6] have been widely used and highly developed because of their outstanding performance and computational efficiency in visual tracking. Compared with traditional learning algorithms such as linear representation model [10], structured output SVM [15] and semisupervised learning [16], these methods learn a correlation filter from a single image patch containing both the target and the background. The correlation filters are trained to accomplish a circular sliding window operation on the training patch on every frame. Heriques et al. proposed the circulant structure with kernels (CSK) by using correlation filters in a kernel space [3], and it was further improved by using histogram of oriented gradients (HOG) features [7], [8] in the kernelized correlation filter (KCF) tracking algorithm [4]. Zhang et al. [14] incorporated context

\footnotetext{
Manuscript received March 2, 2016.

Manuscript revised August 28, 2016.

Manuscript publicized October 4, 2016.

${ }^{\dagger}$ The authors are with the College of Command Information Systems, PLA University of Science and Technology (PLAUST), Nanjing, China.

a) E-mail: emiao_beyond@ $163 . c o m$ (Corresponding author) DOI: 10.1587/transinf.2016EDL8053
}

information into filter learning. $\mathrm{Li}$ et al. [11] employed part-based tracking strategy to visual tracking. Danelljan et al. presented the discriminative scale space tracker (DSST) [6] to cope with scale changes efficiently, and then proposed the spatially regularized discriminative correlation filter (SRDCF) tracker [13]. In SRDCF tracker, the correlation filter is trained on a significantly larger set of negative training samples without corrupting the positive samples, which has demonstrated excellent tracking results. These methods try to handle color videos in gray space. This operation, however, is not very reasonable as the color of the target contains a large quantity of characteristic information which can be further extracted and used as the feature in tracking. Other works [9], [12] exploited color attributes in tracking and achieved good performance.

This letter proposes a robust and efficient tracker combined with color features in correlation filter framework. First, different from the existing methods, we extracts not only gray scale information but also color information as the feature maps to compute the maximum response location. And we also analyze the effect of different color features. Second, we modify the label function of the conventional tracker to enhance the positioning accuracy and employ a discriminative correlation filter to handle scale variations. This proposed method is tested and compared with 11 other state-of-the-art tracking methods. And the results show that our method outperforms the other approaches while running at more than 75 frames per second (FPS).

\section{Tracking Components}

Recently, the KCF tracker [4] has reached excellent performance with high speed (258 FPS). Considering its competitive performance and efficiency, we develop our method based on the KCF tracker.

\subsection{The KCF Tracker}

In $\mathrm{KCF}$ tracker, a classifier is trained using a single image patch $\mathrm{x}$ with size $M \times N$, which is centered around the target. $M \times N$ is set to $\beta w \times \beta h$, where $w \times h$ is the size of the target and $\beta$ is the expansion coefficient. KCF considers all cyclic shifts $\mathrm{x}_{m, n},(m, n) \in\{0, \cdots, M-1\} \times\{0, \cdots, N-1\}$, as the training examples for the classifier. They are labeled with a Gaussian function $\mathrm{y}(m, n)$.

The goal of classifier training is to learn a function 
$f(\mathrm{x})=\langle\mathbf{w}, \varphi(\mathrm{x})\rangle$ that minimizes the squared error over all the samples $\mathrm{x}_{m, n}$ and their regression labels $\mathrm{y}(m, n)$. The objective function is

$$
\mathbf{w}=\underset{\mathbf{w}}{\arg \min } \sum_{m, n}\left|\left\langle\varphi\left(\mathrm{x}_{m, n}\right), \mathbf{w}\right\rangle-\mathrm{y}(m, n)\right|^{2}+\lambda\|\mathbf{w}\|^{2},
$$

where $\varphi$ represents the mapping to the Hilbert space induced by the kernel $\kappa$. The inner product of $\mathrm{x}$ and $\mathrm{x}^{\prime}$ is computed as $\left\langle\varphi(\mathrm{x}), \varphi\left(\mathrm{x}^{\prime}\right)\right\rangle=\kappa\left(\mathrm{x}, \mathrm{x}^{\prime}\right)$. The constant $\lambda \geq 0$ is a regularization parameter.

The objective function is minimized as

$$
\mathbf{w}=\sum_{m, n} \alpha(m, n) \varphi\left(\mathbf{x}_{m, n}\right),
$$

and the coefficient $\alpha$ is defined by

$$
A=\mathscr{F}(\alpha)=\frac{\mathscr{F}(\mathrm{y})}{\mathscr{F}\left(\mathbf{k}_{\mathrm{xx}}\right)+\lambda},
$$

where $\mathscr{F}$ denotes the Fourier transform; $\mathbf{k}_{\mathrm{xx}}(m, n)=$ $\kappa\left(\mathrm{x}_{m, n}, \mathrm{x}\right)$ is the output of the kernel function $\kappa$, which is a Gaussian function $\kappa\left(\mathrm{x}, \mathrm{x}^{\prime}\right)=\exp \left(-\frac{\left|\mathrm{x}-\mathrm{x}^{\prime}\right|^{2}}{\sigma^{2}}\right) . \sigma$ is the bandwidth of Gaussian kernel.

For all cyclic shifts, the outputs of $\kappa$ are calculated as

$$
\mathbf{k}_{\mathrm{xx}^{\prime}}=\exp \left(-\frac{1}{\sigma^{2}}\left(\|\mathrm{x}\|^{2}+\left\|\mathrm{x}^{\prime}\right\|^{2}-2 \mathscr{F}^{-1}\left(X^{*} \odot X^{\prime}\right)\right)\right) \text {. }
$$

In (4), $\mathscr{F}^{-1}$ denotes the inverse Fourier transform; $\odot$ is the element-wise product; $X^{*}=\mathscr{F}^{*}(\mathrm{x}), X^{\prime}=\mathscr{F}\left(\mathrm{x}^{\prime}\right)$, here * denotes the complex conjugate.

In KCF tracker, a patch $\mathrm{z}$ with size $M \times N$ is cropped out in the next frame. The confidence score is computed as

$$
\hat{\mathrm{y}}=\mathscr{F}^{-1}\left(\hat{A} \odot K_{\mathrm{z} \hat{\mathrm{x}}}\right) \text {. }
$$

Here $\hat{A}$ denotes the learned classifier coefficients; $\hat{x}$ denotes the learned target appearance. The target location in the new frame is then detected by searching the position with the highest score.

\subsection{Correlation Tracking Combined with Color Features}

Because of the use of the Fourier transform, the computational efficiency is greatly improved and the multi-channel features can be applied to the tracker to improve the tracking accuracy. Let the number of channels be $C$, then the multi-channel features can be concatenated into a vector $\mathbf{x}=\left[\mathrm{x}_{1}, \mathrm{x}_{2}, \cdots, \mathrm{x}_{C}\right]$, and (4) can be modified as follows

$$
\mathbf{k}_{\mathbf{x x}^{\prime}}=\exp \left(-\frac{1}{\sigma^{2}}\left(\|\mathbf{x}\|^{2}+\left\|\mathbf{x}^{\prime}\right\|^{2}-2 \mathscr{F}^{-1}\left(\sum_{c=1}^{C} F_{c}\right)\right)\right) \text {, }
$$

where $F_{c}=\mathscr{F}^{*}\left(\mathrm{x}_{c}\right) \odot \mathscr{F}\left(\mathrm{x}_{c}^{\prime}\right)$.

According to (6), stronger features can be added to the tracker. In our approach, we use not only gray features but also color features (HSI), as shown in Fig. 1.

HOG is one of the most popular features in computer vision, which is confirmed to be effective and efficient. HOG extracts the gradient information from a cell with a range of pixels. As in KCF tracker, we employ the 31 gradient orientation bins variant in our approach, then choose

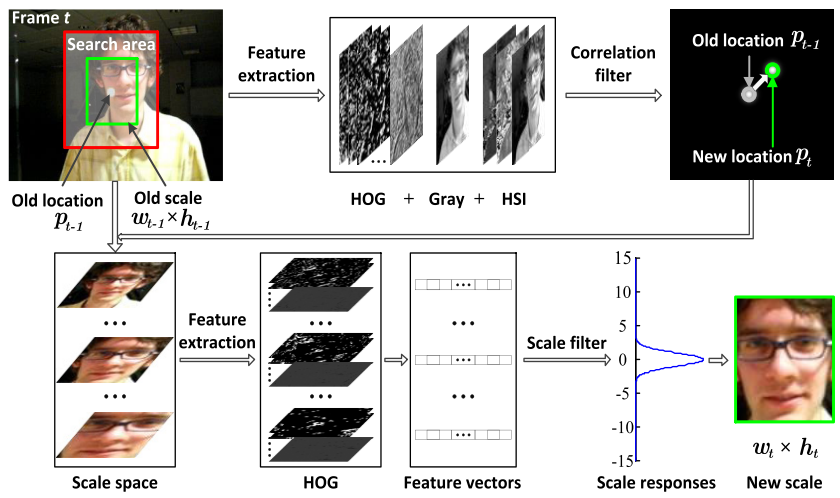

Fig. 1 Flowchart of the proposed tracking algorithm.

normalized HSI as the color feature in our proposed tracker.

Given an RGB image, the hue, saturation and intensity are obtained by

$$
\left\{\begin{array}{l}
H=\left\{\begin{array}{l}
\theta \quad \text { if } B \leq G \\
360-\theta \text { else }
\end{array}\right. \\
S=1-\frac{3}{R+G+B} \min (R, G, B) \\
I=\frac{1}{3}(R+G+B)
\end{array}\right.
$$

with

$$
\theta=\arccos \left\{\frac{\frac{1}{2}[(R-G)+(R-B)]}{\left[(R-G)^{2}+(R-B)(G-B)\right]^{1 / 2}}\right\} .
$$

During training, each sample must be attached a label.

$$
\mathrm{y}(m, n)=\exp \left(-\frac{\left|p-p_{0}\right|^{2}}{2 \sigma_{y}^{2}}\right),
$$

where $\sigma_{\mathrm{y}}$ is spatial bandwidth, $p_{0}=\left(m_{0}, n_{0}\right), p=(m, n)$ and $\left|p-p_{0}\right|=\sqrt{\left(m-m_{0}\right)^{2}+\left(n-n_{0}\right)^{2}}$. At the point $p_{0}$,

$$
\left\{\begin{array}{l}
\left.\mathrm{y}\right|_{p=p_{0}}=\max (\mathrm{y})=1 \\
\left.\frac{\partial \mathrm{y}}{\partial m}\right|_{p=p_{0}}=\left.\frac{\partial \mathrm{y}}{\partial n}\right|_{p=p_{0}}=0
\end{array} .\right.
$$

According to (10), the partial derivatives equal to zero at the maximum point. It means that the responses are too close to be distinguished, so it can not achieve very accurate location, which is verified in Sect. 3.2. Therefore, we modify the label using (11) to reach more accurate location.

$$
\tilde{y}(m, n)=\exp \left(-\frac{\left|p-p_{0}\right|}{2 \rho}\right),
$$

here $\rho$ is a constant that is proportional to target size.

At the point $p_{0}$,

$$
\left\{\begin{array}{l}
\left.\tilde{\mathrm{y}}\right|_{p=p_{0}}=\max (\tilde{\mathrm{y}})=1 \\
\left.\frac{\partial \tilde{\mathrm{y}}}{\partial m}\right|_{\substack{m=m_{0}^{+} \\
n=n_{0}}}=-\frac{1}{2 \rho},\left.\frac{\partial \tilde{\mathrm{y}}}{\partial m}\right|_{\substack{m=m_{0}^{-} \\
n=n_{0}}}=\frac{1}{2 \rho} \\
\left.\frac{\partial \mathrm{y}}{\partial n}\right|_{\substack{n=n_{0}^{+} \\
m=m_{0}}}=-\frac{1}{2 \rho},\left.\frac{\partial \tilde{\mathrm{y}}}{\partial n}\right|_{\substack{n=n_{0}^{-} \\
m=m_{0}}}=\frac{1}{2 \rho}
\end{array} .\right.
$$

Here $m=m_{0}^{+}$and $n=n_{0}^{+}$denote right derivative; $m=m_{0}^{-}$ and $n=n_{0}^{-}$denote left derivative.

In (12), the right and left derivative are inversely proportional to $\rho$, which means that the responses can be distinguished easily. And this is beneficial to locating target as 
Table 1 Comparison of different color features under different labels with the baseline

\begin{tabular}{|c||c|c|c|c|c||c|c|c|c|c||c|}
\hline \multirow{2}{*}{ Evaluation } & \multicolumn{5}{|c||}{ With our label } & \multicolumn{5}{c||}{ With Gaussian label } & \multirow{2}{*}{ KCF } \\
\cline { 2 - 13 } & HSI & LAB & RGB & YCbCr & YUV & HSI & LAB & RGB & YCbCr & YUV & \\
\hline Average DP (\%) & $\mathbf{8 3 . 6}$ & 75.6 & 75.8 & 75.4 & 78 & $\underline{78.8}$ & 73.1 & 75.2 & 72.4 & 74.5 & 71.8 \\
Average OP (\%) & $\mathbf{7 9}$ & 72.9 & $\underline{73.7}$ & 72 & 73.4 & $\underline{73.7}$ & 67.9 & 72.5 & 70.9 & 72.8 & 60.5 \\
\hline
\end{tabular}

Table 2 Comparison with state-of-the-art trackers on the 35 benchmark color sequences

\begin{tabular}{|c||c|c|c|c|c|c|c|c|c|c|c|c|}
\hline Evaluation & Ours & SRDCF & SAMF & RPT & TGPR & DSST & KCF & CN & Struck & CSK & PCOM & STC \\
\hline Average DP (\%) & $\mathbf{8 3 . 6}$ & $\underline{79.8}$ & 77 & 76.4 & 71.4 & 70.9 & 71.8 & 63.8 & 55.6 & 51.5 & 40.4 & 48 \\
Average OP (\%) & $\mathbf{7 9}$ & $\underline{\mathbf{7 3 . 8}}$ & 72.2 & 67.5 & 65.2 & 64.5 & 60.5 & 50.9 & 48.7 & 40 & 33.7 & 31.5 \\
\hline Average speed (FPS) & 78.1 & 7.26 & 18.7 & 5.3 & 0.7 & 41.7 & $\underline{258}$ & 177 & 9.7 & $\mathbf{3 3 8}$ & 24.5 & 150 \\
\hline
\end{tabular}

well as decreasing the location ambiguity in tracking.

To cope with scale variations, we employ a scale pyramid [6] around the target location and learn a discriminative correlation filter for scale estimation as shown in Fig. 1. After finding the target location $p_{t}$ in current frame $t$, Q image patches centered around $p_{t}$ are cropped from the image, and each of size $a^{q} w_{t-1} \times a^{q} h_{t-1}$, where $w_{t-1} \times h_{t-1}$ is the size of the previous frame $t-1, a$ is the scale factor, and $\left.\left.q \in\left\{\mid-\frac{Q-1}{2}\right\rfloor,\left|-\frac{Q-3}{2}\right|, \cdots, \mid \frac{Q-1}{2}\right]\right\}$. Then all the $\mathrm{Q}$ image patches are operated for feature extraction after resizing to the template size, and the features are set to feature vectors for a scale filter. Finally, the highest scale response will be find to estimate the current scale $w_{t} \times h_{t}$ as

$$
\left\{\begin{array}{l}
w_{t}=a^{q^{\prime}} w_{t-1} \\
h_{t}=a^{q^{\prime}} h_{t-1}
\end{array}\right.
$$

where $q^{\prime}$ is the scale number with the highest response.

Finally, the total model parameters are updated frame by frame as

$$
\left\{\begin{array}{l}
\hat{\mathrm{x}}_{t}=(1-\eta) \hat{\mathrm{x}}_{t-1}+\eta \mathrm{z}_{t} \\
\hat{A}_{t}=(1-\eta) \hat{A}_{t-1}+\eta A_{t}
\end{array}\right.
$$

where $\eta$ is the learning rate, $t$ is the index of frame.

\section{Experiments}

We first perform a comprehensive evaluation of our proposed approach under different training labels combined with different color features. And then we compare our approach with 11 state-of-the-art trackers.

\subsection{Experimental Setup}

The tracker is implemented in Matlab 2013a. The experiments are performed on an Intel i5-4690 CPU (3.50 GHz) with 16 GB RAM and 35 challenging color sequences [1] are employed. The expansion coefficient $\beta$, the Gaussian kernel bandwidth $\sigma$ and the regularization parameter $\lambda$ are set to $2.5,0.5$ and $10^{-4}$, which are the same as KCF tracker. The learning rate $\eta$ is set to 0.01 instead of 0.02 in KCF tracker. The scale space number Q, the scale factor $a$ and the parameter $\rho$ used in (11) are set to 27, 1.035 and $\sqrt{w h} / 28$, which are obtained by repeating experiments. Here $w$ and $h$ are the width and height of target.
The results are evaluated by using distance precision (DP) and overlap precision (OP). DP is the percentage of frames where the center location error is smaller than 20 pixels. OP is the percentage of frames where the bounding box overlap is greater than 0.5 .

\subsection{Evaluation of Color Features and Training Labels}

Table 1 shows the results of our proposed method under different training labels combined with different color features including HSI, LAB, RGB, YCbCr and YUV. We use the conventional KCF tracker as a baseline. The first and second highest results are highlighted by bold and underline.

Compared with KCF tracker, our approaches achieve better performance combined with color features and the performance is improved further by using our proposed label instead of Gaussian label. These clearly demonstrate the effectiveness and the robustness of our approach in object tracking. Among the color features in our evaluation, HSI provides the best performance, which significantly outperforms KCF by $18.5 \%$ in average OP and enhances the average DP from $71.8 \%$ to $83.6 \%$. So we choose HSI as the color features in our proposed tracker, as described in Sect. 2.2.

\subsection{Comparison with State-of-the-Art}

We compare our approach with 11 state-of-the-art trackers: CN [9], CSK [3], DSST [6], KCF [4], PCOM [10], RPT [11], SAMF [12], SRDCF [13], STC [14], Struck [15] and TGPR [16]. For fair evaluations, all the trackers are tested under the same experimental conditions. The comparison on the 35 benchmark color sequences is shown in Table 2. We present the results using average DP and OP over all sequences. Moreover, a speed comparison in average FPS is also provided. Like Table 1, the first and second highest results are highlighted by bold and underline.

Among the existing trackers in our evaluation, SRDCF provides the best results with an average DP of $79.8 \%$ and an average OP of $73.8 \%$. our approach exceeds it by achieving an average DP of $83.6 \%$ and an average OP of $79 \%$. In addition, our method is more than 10 times faster than SRDCF in average FPS.

Figure 2 contains the success and precision plots illustrating the mean DP and mean OP over all the 35 color se- 

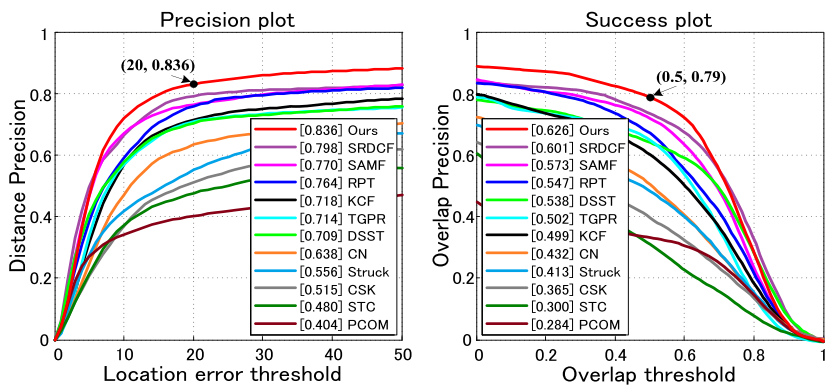

Fig. 2 Precision and success plots. The average DP score at 20 pixels for each tracker is shown in the legend of the precision plot and the area-underthe-curve score for each tracker is reported in the legend of the success plot.

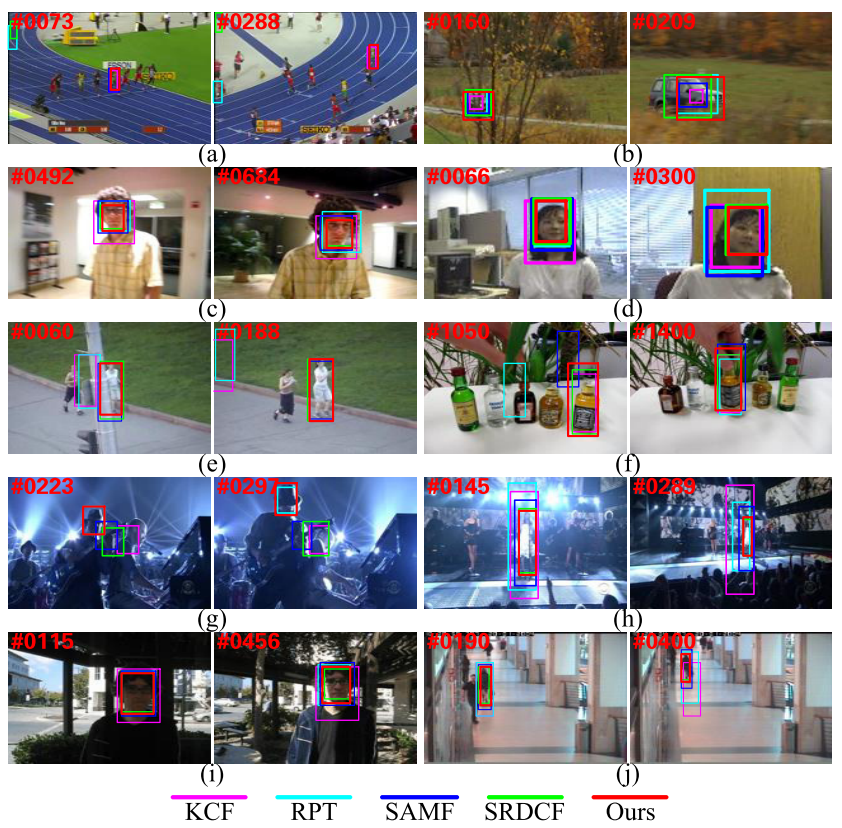

Fig. 3 A visualization of the tracking results of our approach, KCF, RPT, SAMF and SRDCF on 10 benchmark sequences.

quences. In both precision and success plots, our method performs favorably against state-of-the-art trackers. It outperforms SRDCF by $2.5 \%$ and the baseline KCF tracker by $12.7 \%$ in area-under-the-curve score.

Figure 3 shows a qualitative comparison with selected trackers on several benchmark videos. These videos pose challenging problems such as scale changes (Fig. 3(b), (h), and (j)), illumination variations (Fig. $3(\mathrm{c}),(\mathrm{g}),(\mathrm{h})$ and (i)), occlusion (Fig. 3 (b), (e), (f) and (j)), rotation (Fig. 3 (a), (c), (d) and (f)), fast motion (Fig. 3 (b) and (f)), and background clutter (Fig. 3 (f), and (i)). Despite these challenges, our approach obtains the positions of the target accurately.

\section{Conclusion}

To improve the performance of object tracking in color videos, we propose an object tracking method combined with color features. With this method, the color information is dug in tracking. Furthermore, we modify the label function to enhance the positioning accuracy and employ a dis- criminative correlation filter to handle scale variations. On 35 challenging benchmark color sequences, both qualitative and quantitative experiment results show that our approach outperforms state-of-the-art methods.

\section{Acknowledgements}

This work is supported by the National Natural Science Foundation of China (61402519) and the Jiangsu Provincial Nature Science Foundation of China (BK20140071).

\section{References}

[1] Y. Wu, J. Lim, and M.-H. Yang, "Online object tracking: A benchmark," Proc. IEEE Conf. Comput. Vis. Patt. Recog., Portland, USA, pp.2411-2418, June 2013.

[2] B. Guo, and J. Liu, "Real-time tracking with online constrained compressive learning," IEICE Trans. Inf. \& Sys., vol.E96-D, no.4, pp.988-992, April 2013.

[3] J.F. Henriques, R. Caseiro, P. Martins, and J. Batista, "Exploiting the circulant structure of tracking-by-detection with kernels," Proc. European Conf. Comput. Vis., Florence, Italy, pp.702-715, Oct. 2012.

[4] J.F. Henriques, R. Caseiro, P. Martins, and J. Batista, "High-speed tracking with kernelized correlation filters," IEEE Trans. Patt. Anal. Mach. Intell., vol.37, no.3, pp.583-596, March 2015.

[5] T. Liu, G. Wang, Q. Yang, "Real-time part-based visual tracking via adaptive correlation filters," Proc. IEEE Conf. Comput. Vis. Patt. Recog., Boston, USA, pp.4902-4912, June 2015.

[6] M. Danelljan, G. Häger, F.S. Khan, and M. Felsberg, "Accurate scale estimation for robust visual tracking," Proc. British Machine Vis. Conf., Nottingham, UK, pp.1-11, Sept. 2014.

[7] P. Felzenszwalb, R. Girshick, D. McAllester, and D. Ramanan, "Object detection with discriminatively trained part-based models," IEEE Trans. Patt. Anal. Mach. Intell., vol.32, no.9, pp.1627-1645, Sept. 2010.

[8] P. Dollár, R. Appel, S. Belongie, and P. Perona, "Fast feature pyramids for object detection,” IEEE Trans. Patt. Anal. Mach. Intell., vol.36, no.8, pp.1532-1545, Aug. 2014.

[9] M. Danelljan, F.S. Khan, M. Felsberg, and J. van de Weijer, "Adaptive color attributes for real-time visual tracking," Proc. IEEE Conf. Comput. Vis. Patt. Recog., Columbus, USA, pp.1090-1097, June 2014.

[10] D. Wang and H. Lu, "Visual tracking via probability continuous outlier model," Proc. IEEE Conf. Comput. Vis. Patt. Recog., Columbus, USA, pp.3478-3485, June 2014.

[11] Y. Li, J. Zhu, and S.C.H. Hoi, "Reliable patch trackers: Robust visual tracking by exploiting reliable patches," Proc. IEEE Conf. Comput. Vis. Patt. Recog., Boston, USA, pp.353-361, June 2015.

[12] Y. Li and J. Zhu, "A scale adaptive kernel correlation filter tracker with feature integration," Proc. European Conf. Comput. Vis., Zurich, Switzerland, pp.254-265, Sept. 2014.

[13] M. Danelljan, G. Häger, F.S. Khan, and M. Felsberg, "Learning spatially regularized correlation filters for visual tracking," Proc. IEEE Int. Conf. Comput. Vis., Santiago, Chile, pp.4310-4318, Dec. 2015.

[14] K. Zhang, L. Zhang, Q. Liu, D. Zhang, and M.-H. Yang, "Fast visual tracking via dense spatio-temporal context learning," Proc. European Conf. Comput. Vis., Zurich, Switzerland, pp.127-141, Sept. 2014.

[15] S. Hare, A. Saffari, and P.H.S. Torr, "Struck: Structured output tracking with kernels," Proc. IEEE Int. Conf. Comput. Vis., Barcelona, Spain, pp.263-270, Nov. 2011.

[16] J. Gao, H. Ling, W. Hu, and J. Xing, "Transfer learning based visual tracking with Gaussian processes regression," Proc. European Conf. Comput. Vis., Zurich, Switzerland, pp.188-203, Sept. 2014. 